\title{
Decreased expression of miR-9 due to E50K OPTN mutation causes disruption of the expression of BDNF leading to RGC-5 cell apoptosis
}

\author{
BO JIANG ${ }^{1}$, LIN GAO $^{1}$, DAWEI LEI ${ }^{1}$, JIANNAN LIU ${ }^{1}$, ZHENGBO SHAO $^{1}$, XINRONG ZHOU $^{1}$, \\ RENKE $\mathrm{LI}^{2}$, DONGLAI WU ${ }^{3}$, FEI XUE ${ }^{3}$, YUANMAO ZHU ${ }^{3}$ and HUIPING YUAN ${ }^{1}$ \\ ${ }^{1}$ Department of Ophthalmology, The Second Affiliated Hospital, Harbin Medical University, Harbin, Heilongjiang 150086, \\ P.R. China; ${ }^{2}$ Division of Cardiovascular Surgery and Toronto General Research Institute, \\ University Health Network and Department of Surgery, Division of Cardiac Surgery, University of Toronto, \\ Toronto, ON M5G 1L7, Canada; ${ }^{3}$ The Key Laboratory of Veterinary Public Health, Ministry of Agriculture, \\ State Key Laboratory of Veterinary Biotechnology, Harbin Veterinary Research Institute, \\ Chinese Academy of Agricultural Sciences, Harbin, Heilongjiang 150001, P.R. China
}

Received July 25, 2015; Accepted June 14, 2016

DOI: $10.3892 / \mathrm{mmr} .2016 .5810$

\begin{abstract}
The aims of the present study were to investigate the effect of E50K optineurin (OPTN) mutation on RGC-5 cells and to define the role of microRNA-9 (miR-9) in this system. Transfected RGC-5 cells were used to evaluate the effects of E50K OPTN on the expression of miR-9 and subsequent disruption of RGC-5 cell apoptosis was analyzed using western blotting. The results showed that the expression of E50K OPTN was associated with a marked reduction in the levels of miR-9 in the E50K OPTN-transfected RGC-5 cells. The E50K OPTN-dependent reductions in miR-9 led to increased expression of the transcriptional repressor, RE1-silencing transcription factor and decreased the expression of brain-derived neurotrophic factor. Thus, E50K OPTN may disrupt the expression of miR-9, suggesting a potential mechanism by which E50K OPTN mutation may lead to RGC-5 cell apoptosis.
\end{abstract}

\section{Introduction}

A potential role of optineurin (OPTN) when it was first established in 1998 in the GLCE1 region at locus p15-14 on chromosome 10 (1). Our previous study suggested that a novel optineurin genetic mutation encoding a K322E amino acid substitution was associated with primary open-angle

Correspondence to: Dr Huiping Yuan, Department of Ophthalmology, The Second Affiliated Hospital, Harbin Medical University, 246 Xuefu Road, Nangang, Harbin, Heilongjiang 150086, P.R. China

E-mail: yuanhp2013@126.com

Key words: optineurin (E50K), microRNA-9, RE1-silencing transcription factor, brain-derived neurotrophic factor, RGC-5 cells glaucoma in a Chinese family (2). A number of additional disease-causing amino acid substitutions, including E50K, H486R and R545Q have also been confirmed (3-5). The E50K mutation is considered to be the predominant hereditary cause of normal tension glaucoma $(1,3,6)$, and has been found to selectively destroy retinal ganglion cells (RGCs) through oxytosis and apoptosis, whereas other mutants do not have a similar effect (7). Our previous study showed that the mutant E50K optineurin induced the apoptosis of RGCs in transgenic mouse models, however, the precise mechanisms remain to be fully elucidated (8).

Regulation of the expression of brain-derived neurotrophic factor (BDNF) appears central to the mechanism of E50K OPTN-induced RGC apoptosis. Transcription factors, including the repressor, RE1-silencing transcription factor (REST), are essential for the development and function of the nervous system, in part through the regulation of $\operatorname{BDNF}(9,10)$. Increasing the level of endogenous BDNF exerts a neuroprotective effect against tumor necrosis factor- $\alpha$-induced axonal loss (11). The periocular delivery of hydrogels containing Leu-Ile induce the expression of BDNF by increasing the levels of phosphorylated cAMP-responsive element binding protein in the retina, an effect which may enhance the survival of RGCs following optic nerve injury (12). The above findings demonstrate that the expression of BDNF is critical for RGC survival.

Animal genomes encode an abundance of small regulatory RNAs of 22 nucleotides in length (13). These microRNAs (miRNAs) represent a class of gene regulatory molecules in multicellular organisms, which are involved in post-transcriptional regulation through sequence complementarity to the 3' untranslated regions (UTRs) of mRNAs (14). The binding of miRNA to target mRNAs can result in translational repression through mRNA degradation or translational inhibition.

The miRNA, miR-9, is an important regulator of REST, and is considered to contribute to the regulation of the levels of BDNF (9), therefore, miR-9 may be involved in the regulation 
of RGC apoptosis. miR-9 directs the post-transcriptional repression of REST by pairing with conserved sites in the 3' UTR of the REST gene $(15,16)$. A previous investigation provided evidence for a double negative feedback loop between the REST silencing complex and the miRNAs it regulates (17). It was suggested, by software prediction, that miR-9 is the miRNA associated with OPTN (16). These findings suggested that the mutation of E50K OPTN may affect the levels of miR-9, leading to the disruption of REST and subsequent abrogation of the expression of BDNF, which is essential for the survival of RGC-5 cells. The primary aim of the present study was to evaluate whether the expression of E50K OPTN contributes to RGC-5 cell apoptosis through the disruption of miR-9. The present study may aid development of novel ideas for drug development.

\section{Materials and methods}

Cell culture. The RGC-5 cells (American Type Culture Collection, Manassas, VA, USA) were cultured in Dulbecco's modified Eagle's medium (DMEM; Gibco; Thermo Fisher Scientific, Inc., Waltham, MA, USA) supplemented with $10 \%$ heat-inactivated fetal bovine serum (FBS; Hyclone; GE Healthcare Life Sciences) and $1 \%$ penicillin and streptomycin in a humidified $37^{\circ} \mathrm{C}$ incubator with $5 \% \mathrm{CO}_{2}$.

TargetScan. TargetScan (www.targetscan.org/vert_71/) is an online database that matches miRNAs and their target mRNAs. It was used to determine target genes of miR-9.

Plasmid construction. The coding region of the human OPTN cDNA was amplified by PCR using a placental cDNA library (GeneCopoeia, Inc., Rockville, MD, USA) as a template. The reaction mixture $(25 \mu \mathrm{l})$ contained $2.5 \mu \mathrm{l}$ buffer, $2.5 \mu \mathrm{l}$ dNTPs, $2 \mu \mathrm{l}$ cDNA, $0.5 \mu \mathrm{l}$ forward primer, $0.5 \mu \mathrm{l}$ reverse primer, $0.3 \mu \mathrm{l}$ Taq polymerase and $16.7 \mu \mathrm{l}$ double distilled water and the thermocycling conditions were as follows: $95^{\circ} \mathrm{C}$ for $5 \mathrm{~min}$; 30 cycles $95^{\circ} \mathrm{C}$ for $15 \mathrm{sec}, 65^{\circ} \mathrm{C}$ for $30 \mathrm{sec}$ and $72^{\circ} \mathrm{C}$ for $30 \mathrm{sec}$; $72^{\circ} \mathrm{C}$ for $10 \mathrm{~min}$; followed by $4^{\circ} \mathrm{C}$ to stop the reaction. The nucleotide sequence of the cloned OPTN was identical with that reported in GenBank (accession no. NM_021980; www. ncbi.nlm.nih.gov/nuccore/BC032762.1). The PCR product was cloned into the pcDNA3.1 expression vector (Invitrogen; Thermo Fisher Scientific, Inc.) by GeneCopoeia, Inc., which places a His tag in-frame with the 3'-end of the cDNA. Mutations in the OPTN cDNA were created using a PCR-based, site-directed mutagenesis strategy (also conducted by GeneCopoeia, Inc.) to introduce nucleotide changes encoding the E50K amino acid substitution. The nucleotide sequences of the mutant E50K and wild-type OPTN cDNA constructs were confirmed using automated DNA sequencing.

RNA interference and plasmid transfection. The mmu-miR-9-5p (accession no. MIMAT0000142) mimic/inhibitor and negative control/inhibitor (GenePharma, Shanghai, China) were dissolved in RNase-free $\mathrm{H}_{2} \mathrm{O}$. The cells were plated in 6 -well plates $\left(2 \times 10^{5}\right.$ cells/well) for $18-24 \mathrm{~h}$ at $37^{\circ} \mathrm{C}$ in $5 \% \mathrm{CO}_{2}$ prior to transfection. The cells were co-transfected with the miRNA and expression plasmids using Lipofectamine 2000 (Invitrogen; Thermo Fisher
Scientific, Inc.), according to the manufacturer's protocol, when the cells were $\sim 70 \%$ confluent. RNA-lipid complexes were introduced into each well of cells $(4 \mu \mathrm{l} /$ well for mimic and duplex for inhibitor), with $4 \mu \mathrm{g}$ /well plasmids. Between 48 and $72 \mathrm{~h}$ at $37^{\circ} \mathrm{C}$ in $5 \% \mathrm{CO}_{2}$ post-transfection, the levels of target genes were assessed using reverse transcription-quantitative polymerase chain reaction (RT-qPCR) and western blot analyses.

Analysis of mRNA and miRNA expression. The expression levels of mRNA and miRNA were determined using RT-qPCR analysis. Total RNA was extracted from the transfected cells using TRIzol reagent (Invitrogen; Thermo Fisher Scientific, Inc.). The RT-qPCR analysis for miRNAs was performed using an All-in-One ${ }^{\mathrm{TM}}$ miRNA qPCR detection kit (GeneCopoeia, Inc., Rockville, MD, USA) and SYBR ${ }^{\circledR}$ Premix Ex Taq II (Takara Bio, Inc., Otsu, Japan) for coding genes on a Lightcycler 480 system (Roche, Mannheim, Germany), according to the manufacturer's protocol. The thermocycling conditions were as follows: $95^{\circ} \mathrm{C}$ for $10 \mathrm{~min}$; followed by 40 cycles of $95^{\circ} \mathrm{C}$ for $10 \mathrm{sec}, 62^{\circ} \mathrm{C}$ for $20 \mathrm{sec}$ and $72^{\circ} \mathrm{C}$ for $20 \mathrm{sec}$. Ratios to indicate the relative quantities were automatically exported by the Lightcycler 480 version 1.5.0 software.

Western blot analysis. The total protein was extracted from the cultured RGC-5 cells. Samples were homogenized at $4^{\circ} \mathrm{C}$ in lysis buffer, containing $50 \mathrm{mM}$ Tris (pH 8.0), $150 \mathrm{mM} \mathrm{NaCl}, 50 \mathrm{mM}$ EDTA, $0.5 \%$ sodium deoxycholate and $1 \%$ Triton $\mathrm{X}-100$, containing a protease inhibitor cocktail (Sigma-Aldrich, St. Louis, MO, USA), 2 mM DTT and $0.1 \mathrm{mM}$ PMSF. Protein content was determined using a Bio-Rad DC protein assay (Bio-Rad Laboratories, Inc., Hercules, CA, USA). Subsequently, $50 \mu \mathrm{g}$ total protein was resuspended in loading buffer (20\% glycerol, $10 \%$ SDS and $0.1 \%$ bromophenol blue), incubated for $5 \mathrm{~min}$ at $95^{\circ} \mathrm{C}$ and loaded onto a $10 \%$ polyacrylamide gel (Invitrogen; Thermo Fisher Scientific, Inc.). Following electrophoresis, protein was transferred onto a PVDF membrane and blocked in 5\% nonfat milk. The membrane was then incubated overnight at $4^{\circ} \mathrm{C}$ with rabbit anti-BDNF antibody (1:500; Santa Cruz Biotechnology, Inc., Santa Cruz, CA, USA; cat. no. sc-20981), rabbit anti-REST antibody (1:1,000; Abcam, Hong, China; cat. no. ab21635) or mouse anti- $\beta$-actin antibody $(1: 1,000$; Beyotime Institute of Biotechnology, Shanghai, China; cat. no. AA128) as an internal control. Goat horseradish peroxidase (HRP)-conjugated anti-rabbit (cat. no. ZB2308) and anti-mouse (cat. no. ZB2305) secondary antibodies (1:2,000; Zhongshan Goldenbridge Biotechnology Co., Ltd., Beijing, China) was added to the membrane for $2 \mathrm{~h}$ at room temperature. The blots were washed several times with saline buffer (Tris-buffered saline with Tween-20 (25 mM Tris-HCl, $150 \mathrm{mM} \mathrm{NaCl}$ and $0.1 \%$ Tween-20), incubated with ECL (HRP; $100 \mu \mathrm{ECL} / \mathrm{cm}^{2}$ of membrane; Pierce Biotechnology, Inc., Rockford, IL, USA) for $1 \mathrm{~min}$ at room temperature, and evaluated using a LAS-3000 luminescent image analyzer (Fujifilm; Tokyo, Japan). Band intensity was measured using Image J software version 2.1.4.7 (imagej.nih.gov/ij).

Statistical analysis. All data are presented as the mean \pm standard deviation of at least three independent experiments, each 


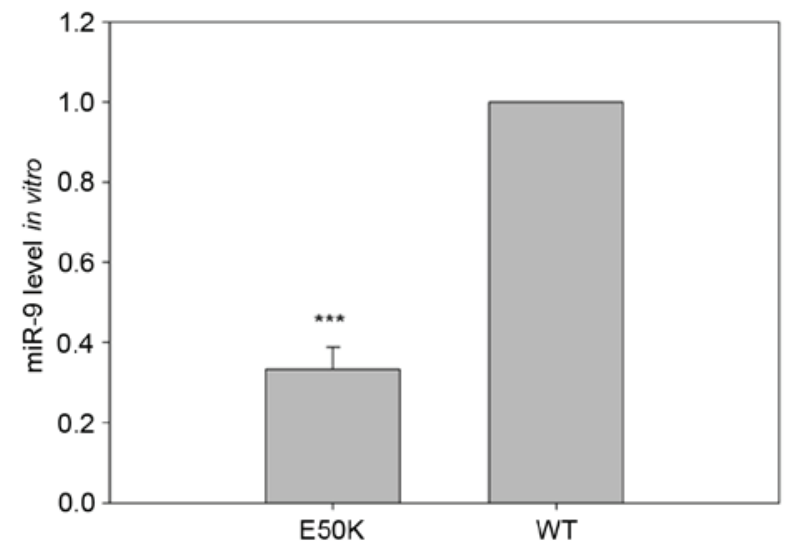

Figure 1. E50K OPTN reduces the expression of miR-9 in RGC-5 cells. Reverse transcription-quantitative polymerase chain reaction analysis was used to evaluate the expression levels of miR-9 in total RNA isolated from RGC-5 cells transfected with wild-type and E50K OPTN. Data are presented as the mean \pm standard error of the mean of triplicate experiments ${ }^{* * *} \mathrm{P}<0.001$ vs. the WT group. OPTN, optineurin; miR, microRNA; WT, wild-type.

performed in triplicate. Statistical evaluation was performed using an unpaired Student's $t$-test. ${ }^{*} \mathrm{P}<0.05$ was considered to indicate a statistically significant differences. Calculations were performed using standard statistical software (SigmaPlot; Systat Software, Inc, San Jose, CA, USA).

\section{Results}

E50K OPTN decreases the expression of miR-9 in transfected $R G C-5$ cells. The present study first evaluated the effect of the expression of mutant E50K OPTN on the mRNA levels of miR-9. The levels of miR-9 were measured using RT-qPCR analysis following the isolation of total RNA from the transfected RGC-5 cells. The transfection-mediated overexpression of E50K OPTN in the RGC-5 cells led to a statistically significant decrease in the levels of miR-9, compared with the RGC-5 cells transfected with a construct overexpressing wild-type OPTN ( $\mathrm{P}<0.001$; Fig. 1). The reduced expression of miR-9 due to E50K OPTN suggested a potential role of miR-9 in RGC-5 cells.

Expression of REST is increased in E50K OPTN-transfected $R G C-5$ cells. miR-9 is known to regulate the expression of the transcription factor, REST, and it has been previously shown that the transfection of miR-9 significantly decreased the activity of a reporter construct driven by the 3' UTR of REST (17). As overexpression of E50K OPTN significantly reduced the expression levels of miR-9, the present study subsequently evaluated the levels of REST in RGC-5 cells transfected to overexpress E50K OPTN. The transfection-mediated expression of E50K OPTN led to a modest increase in the mRNA levels of REST in the mock-treated RGC-5 cells, although this difference was not statistically significant (Fig. 2A). Consistent with previous studies, the in vitro transfection of RGC-5 cells with miR-9 mimic led to a marked reduction in the mRNA levels of REST, whereas the introduction of an miR-9 mimic inhibitor led to modest increases in the mRNA levels of REST. Evaluation of the
A

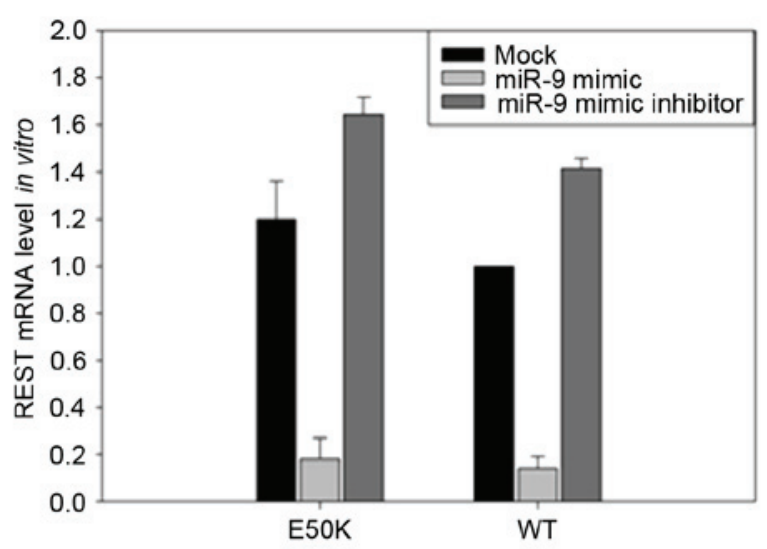

B

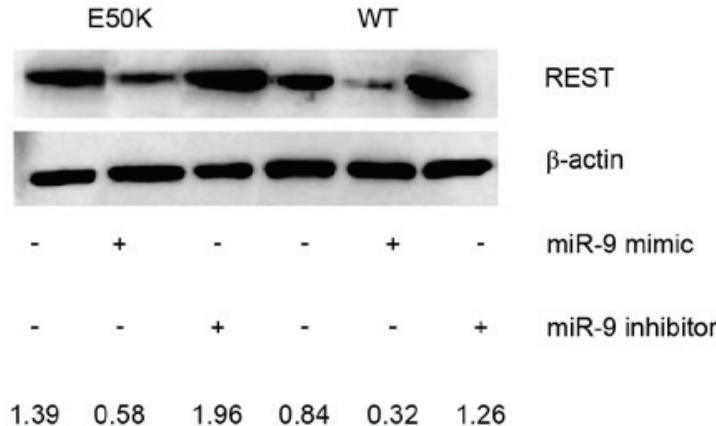

Figure 2. E50K OPTN increases the expression of REST in RGC-5 cells. Expression of REST in RGC-5 cells co-transfected with either an E50K OPTN or WT OPTN expression vector and an miR-9 mimic oligonucleotide, miR-9 mimic inhibitor oligonucleotide, or mock oligonucleotide. Expression levels were measured using (A) reverse transcription-quantitative polymerase chain reaction and (B) western blot analyses with ratio of target protein to $\beta$-actin indicated. OPTN, optineurin; REST, RE1-silencing transcription factor; miR, microRNA; WT, wild-type.

protein levels of REST in the transfected RGC-5 cells closely correlated with the mRNA levels of REST mRNA (Fig. 2B). The protein levels of REST were markedly increased in the mutant E50K OPTN models, compared with the wild-type RGC-5 cells (Fig. 2B).

Expression of BDNF is decreased in E50K OPTN-transfected $R G C-5$ cells. Our previous study suggested that BDNF is essential for the survival of RGC-5 cells (18). Therefore, the present study compared the levels of BDNF in E50K OPTN the RGC-5 cells transfected to express wild-type OPTN. The mock-treated RGC-5 cells transfected to express E50K OPTN exhibited decreased mRNA levels of BDNF, compared with mock-treated RGC-5 cells transfected with wild-type OPTN $\left({ }^{* * *} \mathrm{P}<0.001 ; \mathrm{n}=3\right)$, as shown in Fig. 3A. Co-transfection with the miR-9 mimic led to increased mRNA expression levels of BDNF in the E50K and wild-type OPTN RGC-5 cells, whereas treatment with the miR-9 mimic inhibitor led to decreased mRNA levels of BDNF, compared with the mock-treated cells (Fig. 3A). Evaluation of the protein levels of BDNF in the E50K transfected RGC-5 cells showed that the protein expression levels were also attenuated, compared with the wild-type controls, and exhibited patterns that correlated closely with the mRNA levels of BDNF (Fig. 3B). 

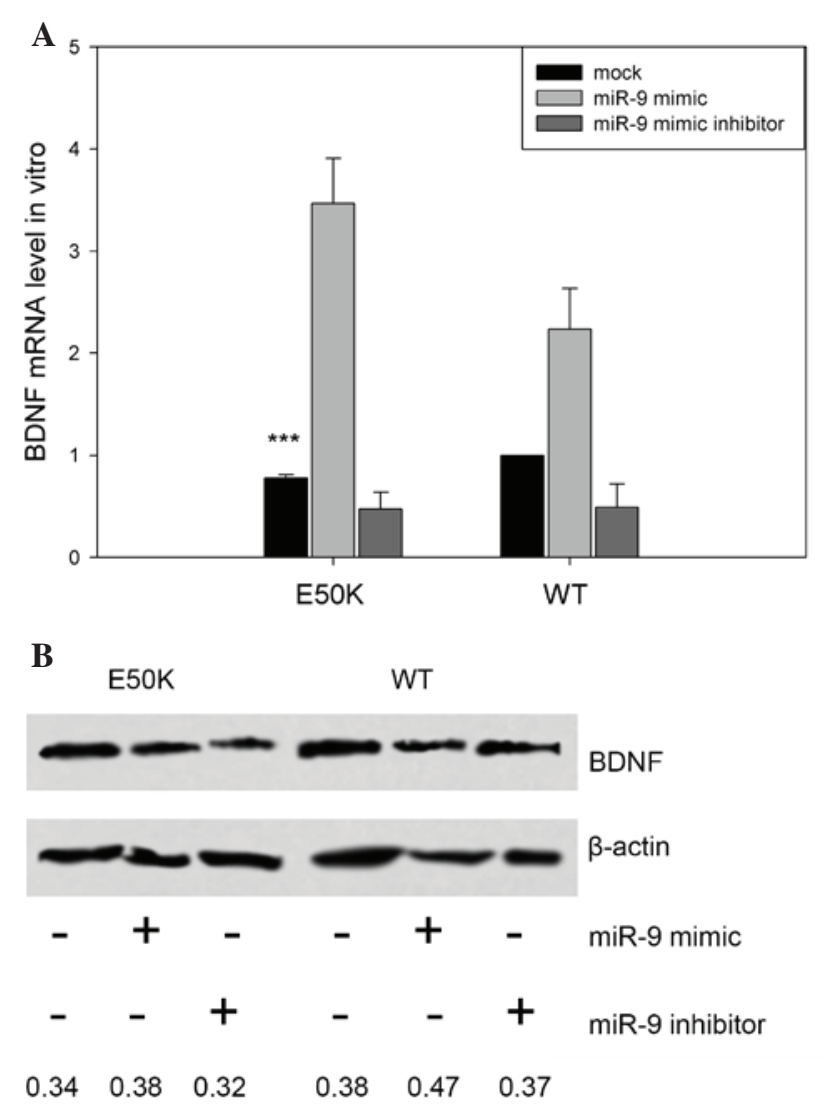

Figure 3. E50K OPTN decreases the expression of BDNF in RGC-5 cells. Expression of BDNF in RGC-5 cells co-transfected with either an E50K OPTN or wild-type OPTN expression vector and an miR-9 mimic oligonucleotide, miR-9 mimic inhibitor oligonucleotide or mock oligonucleotide. Expression levels were measured using (A) reverse transcription-quantitative polymerase chain reaction and $(\mathrm{B})$ western blot analyses with ratio of target protein to $\beta$-actin indicated. ${ }^{* * *} \mathrm{P}<0.001$. OPTN, optineurin; BDNF, brain-derived neurotrophic factor miR, microRNA; WT, wild-type.

\section{Discussion}

The results of the present study showed that the E50K OPTN mutation is associated with markedly reduced expression levels of miR-9 using the cultured RGC-5 cells. E50K OPTN-dependent reductions in miR-9 led to increased expression of the transcriptional repressor, REST, suggesting a potential mechanism by which the E50K OPTN mutation may lead to increased RGC-5 cell apoptosis.

The transcriptional repression of miR-9 by REST has been suggested. The present study provided evidence that the E50K OPTN mutation downregulated the expression of miR-9 in RGC-5 cell models (Fig. 1). miR-9 has been implicated in development of the nervous system, and in physiological and pathological processes in several organisms (19).

miRNAs can also bind to protein factors and recruit them to specific mRNAs to indirectly control the binding of other regulatory factors (13). TargetScan prediction of miRNA targets identified REST as a target gene of miR-9. The results of the present study directly demonstrate a repressive function of miR-9 on the expression of REST (Fig. 2), consistent with a previous report, which suggested a network of REST and miRNAs in neuronal gene expression (9).
A series of studies have confirmed that BDNF prevents the apoptotic death of neurons during development (20-22). Caspase inhibitors can support neurite regeneration by preventing RGC apoptosis and allowing the rescue of adult neurons of the central nervous system, and BDNF may have a protective effect against apoptosis in addition to promoting neurite regeneration by the surviving cells (20). The analysis of polymorphisms of the gene has revealed a statistically significant association of BDNF with the risk of RGC apoptosis (21). BDNF results in the activation of pro-survival cell-signaling pathways, which can afford neuroprotection to the retina (22). REST act to coordinate neuronal gene expression and promote neuronal identity, in part through $\operatorname{BDNF}(9)$.

The cells used in the present study were those of the RGC-5 line, and these are reported to be the same as the $661 \mathrm{~W}$ cell line, which is reported to be of cone photoreceptor origin. A prior investigation also used HEK 293 cells to examine the expression of miR-9, and the regulation of REST and BDNF, however, the difference between the miR-9 mimic group and inhibitor group were not statistically significant, suggesting that this mechanism may be specific for diseases of the nervous system.

In conclusion, the E50K OPTN mutation markedly reduced the levels of miR-9, which led to alterations in REST and reduced expression levels of BDNF in RGC-5 cells.

\section{Acknowledgements}

The present study was funded by the National Natural Science Foundation of China (grant no. 81271000) and the Major State Basic Research Development Program of China (973 Program; grant no. 2011CB707502). The current study was directed by Professor Renke Li of the Division of Cardiovascular Surgery and Toronto General Research Institute, University Health Network and Department of Surgery, Division of Cardiac Surgery, University of Toronto (Toronto, Canada).

\section{References}

1. Rezaie T, Child A, Hitchings R, Brice G, Miller L, Coca-Prados M, Héon E, Krupin T, Ritch R, Kreutzer D, et al: Adult-onset primary open-angle glaucoma caused by mutations in optineurin. Science 295: 1077-1079, 2002.

2. Xiao Z, Meng Q, Tsai JC, Yuan H, Xu N and Li Y: A novel optineurin genetic mutation associated with open-angle glaucoma in a Chinese family. Mol Vis 15: 1649-1654, 2009.

3. Aung T, Rezaie T, Okada K, Viswanathan AC, Child AH, Brice G, Bhattacharya SS, Lehmann OJ, Sarfarazi M and Hitchings RA: Clinical features and course of patients with glaucoma with the E50K mutation in the optineurin gene. Invest Ophthalmol Vis Sci 46: 2816-2822, 2005.

4. Leung YF, Fan BJ, Lam DS, Lee WS, Tam PO, Chua JK, Tham CC, Lai JS, Fan DS and Pang CP: Different optineurin mutation pattern in primary open-angle glaucoma. Invest Ophthalmol Vis Sci 44: 3880-3884, 2003.

5. Fuse N, Takahashi K, Akiyama H, Nakazawa T, Seimiya M, Kuwahara S and Tamai M: Molecular genetic analysis of optineurin gene for primary open-angle and normal tension glaucoma in the Japanese population. J Glaucoma 13: 299-303, 2004.

6. Alward WL, Kwon YH, Kawase K, Craig JE, Hayreh SS, Johnson AT, Khanna CL, Yamamoto T, Mackey DA, Roos BR, et al: Evaluation of optineurin sequence variations in 1,048 patients with open-angle glaucoma. Am J Ophthalmol 136: 904-910, 2003 
7. Chalasani ML, Swarup G and Balasubramanian D: Optineurin and its mutants: Molecules associated with some forms of glaucoma. Ophthalmic Res 42: 176-184, 2009.

8. Chi ZL, Akahori M, Obazawa M, Minami M, Noda T, Nakaya N, Tomarev S, Kawase K, Yamamoto T, Noda S, et al: Overexpression of optineurin E50K disrupts Rab8 interaction and leads to a progressive retinal degeneration in mice. Hum Mol Genet 19: 2606-2615, 2010.

9. Wu J and Xie X: Comparative sequence analysis reveals an intricate network among REST, CREB and miRNA in mediating neuronal gene expression. Genome Bio 7: R85, 2006.

10. Koch JM, Hinze-Selch D, Stingele K, Huchzermeier C, Goder R, Seeck-Hirschner M and Aldenhoff JB: Changes in CREB phosphorylation and BDNF plasma levels during psychotherapy of depression. Psychother Psychosom 78: 187-192, 2009.

11. Fujino H, Kitaoka Y, Hayashi Y, Munemasa Y, Takeda H, Kumai T, Kobayashi S and Ueno S: Axonal protection by brain-derived neurotrophic factor associated with CREB phosphorylation in tumor necrosis factor-alpha-induced optic nerve degeneration. Acta Neuropathol 117: 75-84, 2009.

12. Nakatani M, Shinohara Y, Takii M, Mori H, Asai N, Nishimura S, Furukawa-Hibi Y, Miyamoto Y and Nitta A: Periocular injection of in situ hydrogels containing Leu-Ile, an inducer for neurotrophic factors, promotes retinal ganglion cell survival after optic nerve injury. Exp Eye Res 93: 873-879, 2011.

13. Ambros V: microRNAs: Tiny regulators with great potential. Cell 107: 823-826, 2001.

14. Xiao F, Zuo Z, Cai G, Kang S, Gao X and Li T: miRecords: An integrated resource for microRNA-target interactions. Nucleic Acids Res 37 (Database issue): D105-D110, 2009.
15. Jiang Q, Wang Y, Hao Y, Juan L, Teng M, Zhang X, Li M, Wang G and Liu Y: miR2 Disease: A manually curated database for microRNA deregulation in human disease. Nucleic Acids Res 37 (Database issue): D98-D104, 2009.

16. Friedman RC, Farh KK, Burge CB and Bartel DP: Most mammalian mRNAs are conserved targets of microRNAs. Genome Res 19: 92-105, 2009.

17. Packer AN, Xing Y, Harper SQ, Jones L and Davidson BL The bifunctional microRNA miR-9/miR-9* regulates REST and CoREST and is downregulated in Huntington's disease. J Neurosci 28: 14341-14346, 2008.

18. Yang B, Shan L, Song W, Xiao Z, Shi L and Yuan H: Copolymer-1 immunization reduces damage in retinal ganglion cells under high intraocular pressure through altering the expression of retinal neurotrophins. J Ocul Pharmacol Ther 26: 11-19, 2010.

19. Leucht C, Stigloher C, Wizenmann A, Klafke R, Folchert A and Bally-Cuif L: MicroRNA-9 directs late organizer activity of the midbrain-hindbrain boundary. Nat Neurosci 11: 641-648, 2008.

20. Oshitari T and Adachi-Usami E: The effect of caspase inhibitors and neurotrophic factors on damaged retinal ganglion cells. Neuroreport 14: 289-292, 2003.

21. Nowak A, Majsterek I, Przybyłowska-Sygut K, Pytel D, Szymanek K, Szaflik J and Szaflik JP: Analysis of the expression and polymorphism of APOE, HSP, BDNF, and GRIN2B genes associated with the neurodegeneration process in the pathogenesis of primary open angle glaucoma. Biomed Res Int 2015: 258281, 2015

22. Gupta V, You Y, Li J, Golzan M, Klistorner A, van den Buuse M and Graham S: BDNF impairment is associated with age-related changes in the inner retina and exacerbates experimental glaucoma. Biochim Biophys Acta 1842: 1567-1578, 2014. 